\title{
El cuerpo y sus reconfiguraciones: relatos de mujeres con cáncer de mama
}

\author{
Carmen Rodríguez-Reinado, Ana Delgado, y Juan Alguacil \\ Universidad de Huelva (España)
}

\begin{abstract}
El cuerpo es una construcción social sujeta a múltiples representaciones que dotan de sentido y significado su morfología, y cambiante en función del contexto histórico, social y cultural. Las mujeres que padecen cáncer de mama experimentan grandes cambios en sus cuerpos durante el tratamiento quirúrgico y farmacológico, que no solo conllevan a consecuencias físicas sino también psicológicas y sociales. En este sentido los objetivos del estudio fueron: 1). Comprender el proceso de configuración y reconfiguración del cuerpo durante la enfermedad en las mujeres con cáncer de mama. 2). Analizar las vivencias de las mujeres respecto a las alteraciones del cuerpo durante el tratamiento del cáncer de mama. La metodología empleada para la consecución de los objetivos fue cualitativa basada en entrevistas semiestructuradas a mujeres con cáncer de mama. Los relatos de las mujeres ponen de manifiesto consecuencias psicológicas y sociales por los cambios que se producen en sus cuerpos a raíz del tratamiento. Lo cual evidencia la imperiosa necesidad de abordar este aspecto por el equipo de profesionales sanitario en el proceso asistencial del tratamiento de cáncer de mama.
\end{abstract}

Palabras clave: Cáncer de mama, representación social, cuerpo, metodología cualitativa, imagen corporal.

The body and its reconfigurations: stories of women with breast cancer Stories of women with breast cancer. The body is a social construction subject to multiple representations. These representations give sense and significance to its morphology and change in line with historical, social and cultural contexts. The bodies of women with breast cancer undergo important changes in the course of their treatment. These changes have not only physical consequences but also psychological and social ones. Study objectives: (1) to understand the process of the body's configuration and reconfiguration in the period of illness in women with breast cancer; (2) to analyse these women's experiences of the changes in their bodies during their treatment for breast cancer. Methodology adopted: qualitative, based on semi-structured interviews with women with breast cancer. Findings: the women's accounts reveal psychological and social consequences stemming from the changes brought about in their bodies by their treatment. Conclusion: the study evidences the compelling need for the team of health professionals to address the body in the caring process of breast cancer treatment.

Keywords: Breast cancer, social representation, body, qualitative methodology, body image.

\footnotetext{
Correspondencia: Ana Delgado Parrilla. Universidad de Huelva, Departamento de Sociología, Trabajo Social y Salud Pública. Facultad de Trabajo Social. Avda. de las Fuerzas Armadas, s/n. C.P.: 21007. Huelva (España). E-mail: ana.delgado@dstso.uhu.es
} 
El cuerpo es una construcción social sujeta a múltiples representaciones que dotan de sentido y significado su morfología, y cambiante en función del contexto histórico, social y cultural. Por ejemplo, en las sociedades tradicionales de tipo comunitario, sujeto y cuerpo no son elementos separados entre sí, sino que se entienden como una unidad que forma parte de la naturaleza, del cosmos, de la comunidad. Sin embargo, en las sociedades más individualistas, en las sociedades modernas, el cuerpo se entiende como un elemento aislado de la persona, donde este se define como frontera, como diferenciación entre el yo y los otros, al mismo tiempo que se produce un proceso de desvinculación con la naturaleza (Le Breton, 1990).

En la actualidad, pervive esta concepción del cuerpo como «factor de individuación» (Durkheim, 1893, 1987), pero surgen nuevos significados. El desarrollo del capitalismo ha traído consigo una diversificación de los modos de vida, una atomización de los sujetos y una pérdida de arraigo con la comunidad, que hacen que el sujeto se repliegue sobre sí mismo y tome a su cuerpo como un lugar de reconciliación y reconquista de una y uno mismo. Es una marca personal que nos diferencia «del otro» (Simmel, 1908, 1927) pero también se vuelve un refugio, un espacio de búsqueda de certezas en una sociedad gobernada por la incertidumbre (Le Breton, 1990). Por tanto, el cuerpo se establece como el territorio propio que interacciona relacionalmente con otras personas y con el contexto social, cultural y político y que, por tanto, se construye a partir de esta interacción entre las distintas esferas; adquiriendo representaciones y significados simbólicos en cuanto a su forma, finalidad o función (Figueroa, 2018).

Imagen corporal y cáncer de mama: cambios y estrategias reconfiguratorias

La imagen corporal constituye la representación que la persona hace de sí misma en relación con su cuerpo, reuniendo los atributos físicos que lo configuran y formulando un juicio social al confrontar su autopercepción con la percepción del cuerpo ideal donde se inscriben los cánones de belleza y salud (Figueroa, Valadez, Rivera, y Montes, 2017); así como la mirada del otro. Si no existe concordancia entre la autopercepción y los estándares ideales, la imagen corporal puede ser fruto de insatisfacción y ansiedad (Przezdziecki et al., 2013).

Algunas investigaciones (Belber-Gómez, Valencia-Agudo, y De la Viuda-Suárez, 2018; Biglia et al., 2010; Coll-Planas, 2014; Coll-Planas y Visa 2015; Figueroa-Varela, 2018; Núñez et al., 2018; Przezdziecki et al., 2013; Rodríguez, Blasco, Vargas, y Benito, 2011; Rodrigues, Ribeiro, y Tavares, 2012; Teo et al., 2016) han estudiado la influencia que tienen los cambios provocados en el cuerpo por el tratamiento del cáncer de mama en la imagen corporal, el deseo o la sexualidad y la interacción con el entorno de las mujeres que lo padecen. Los principales hallazgos muestran que el cáncer de mama es una enfermedad cuyo tratamiento (cirugía, quimioterapia, radioterapia y hormonoterapia) amenaza zonas como los senos o el 
cabello que son portadoras de un significado simbólico que está muy relacionado con la identidad social del género femenino. Los cambios que se producen en estas zonas del cuerpo acaban afectando a la imagen corporal que construyen las mujeres de sí mismas (Belber-Gómez et al., 2018; Núñez et al., 2018).

Ahora bien, estos cambios en la imagen corporal no sólo modifican la relación que las mujeres establecen con sus cuerpos, sino también el vínculo con «el otro» (Simmel, 1908/1927). Así mismo, también puede dar lugar a un cuestionamiento de su identidad como mujer, dado que la corporeidad está definida por el rol social de reproducción (Figueroa, 2018) y la mirada masculina en el sistema sexo/género hegemónico. Por tanto, el diagnóstico de cáncer de mama constituye un evento estresante que tiene repercusiones no sólo en el estado físico, sino también en el estado psicológico de la paciente, pues se enfrenta a una nueva configuración de su corporeidad, pudiendo esto desembocar en estados de estrés, ansiedad y depresión (Núñez et al., 2018).

Por otro lado, otros estudios han puesto de manifiesto que la experiencia que las mujeres viven con su corporeidad durante la enfermedad puede llevar a entender el cuerpo como un elemento disociado, ajeno, fragmentado y extraño (Frank, 1995; Le Breton, 1991). Como apunta Coll-Planas (2015) la comunicación del diagnóstico positivo de cáncer de mama activa esta estrategia de disociación con el cuerpo, aunque también se observa en algunas mujeres la tendencia opuesta hacia una reconexión y una mayor escucha activa para con sus cuerpos.

En la presente investigación, se adopta una perspectiva del estudio de «lo cotidiano centrado en el cuerpo» (Le Breton, 1990), sobre las vivencias de las mujeres que han padecido cáncer de mama. Dicha perspectiva permite comprobar las repercusiones de las interacciones del sujeto con el mundo; entendiendo lo cotidiano como "una pasarela entre el mundo controlado y tranquilo de cada uno y las incertidumbres y el aparente desorden de la vida social" (Le Breton, 1990, p.92). Con base a este enfoque se plantearon como objetivos de investigación:

1. Comprender el proceso de configuración y reconfiguración del cuerpo durante la enfermedad en las mujeres con cáncer de mama.

2. Analizar las vivencias de las mujeres respecto a las alteraciones del cuerpo durante el tratamiento del cáncer de mama.

\section{MÉTODO}

La estrategia metodológica aplicada fue cualitativa. Este tipo de metodología ha resultado especialmente válida para la entender los factores que afectan a la salud, así como la toma de decisiones estratégicas en la planificación de servicios sanitarios (Rodríguez et al., 2011). El ámbito del estudio fue provincial: Huelva. 


\section{Participantes}

La muestra aplicada fue de tipo intencional. Los criterios de inclusión muestral fueron: 1) Haber sido diagnosticada de cáncer de mama en un periodo no superior de 10 años. 2) Mujeres que residen en la provincia de Huelva. 3) Mujeres que tengan la nacionalidad española.

\section{Instrumentos}

Se aplicó la entrevista individual semiestructurada como técnica de recogida de información. Se diseñó un guion de tema con las dimensiones de análisis pertinentes a los objetivos de investigación. El guion de tema fue pilotado en una pequeña muestra de mujeres para testar su comprensión y validez. El formato de las preguntas del guion fue abierto. En total se realizaron 11 entrevistas.

\section{Procedimiento}

La estrategia de captación de las personas se llevó a cabo a través del personal sanitario del hospital Juan Ramón Jiménez. Antes de realizar las entrevistas las mujeres firmaron el consentimiento informado sobre los objetivos y metodología del estudio.

\section{Análisis de datos}

La información recabada de las entrevistas fue transcrita literalmente. Se realizó un control de calidad de las transcripciones. Una vez transcritas las entrevistas, la información fue introducida en el programa Atlas.Ti para proceder a su codificación y posterior análisis de contenido.

\section{RESULTADOS}

En términos generales, las narrativas de las mujeres entrevistadas están envueltas por sentimientos dicotómicos de pérdida y resiliencia. Por un lado, cuentan cómo el cambio producido en su apariencia física a consecuencia del tratamiento del cáncer de mama generó en ellas sensaciones de inseguridad, miedo e insatisfacción. En este sentido, se pone de manifiesto distintas estrategias de reconfiguración de su imagen corporal para paliar los efectos de dicha pérdida. Así mismo, las mujeres revelan en sus relatos la importancia del entorno en el proceso de aceptación de su nueva corporeidad.

Con base a este primer análisis realizado, hemos organizado los resultados en torno a tres dimensiones: la experiencia subjetiva, la interacción y las prácticas reconfiguratorias. 


\section{El cuerpo y la dimensión de la experiencia subjetiva}

Se distinguen tres momentos diferentes durante el proceso de la enfermedad que explican las vivencias de las mujeres con cáncer de mama respecto a los cambios corporales que han tenido que hacer frente durante el tratamiento: antes, durante y tras el mismo.

\section{Antes del tratamiento}

Los discursos de las mujeres evidencian que la primera vivencia respecto a sus cuerpos en relación a la enfermedad se sitúa en el momento en que se le comunica un diagnóstico positivo de cáncer de mama. En relación a ello, todas las mujeres entrevistadas, emplean un lenguaje agresivo para expresar sus emociones en relación al momento del diagnóstico o de la operación. De ahí que sean recurrentes los verbos "cortar" o "quitar" para referirse a la intervención quirúrgica de mastectomía radical. Tan solo una de las mujeres entrevistadas se refiere a dicha experiencia como "me operaron el pecho".

Los relatos de las mujeres evocan sentimientos de pérdida: "me sentía incompleta". Tanto es el sentimiento de pérdida que en muchos relatos el lenguaje de las mujeres parece adquirir las connotaciones de un robo, de un robo de su cuerpo, en tanto en cuanto se produce una intrusión del sistema sanitario, una apropiación de sus cuerpos, dejando de ser propios para pasar a ser meros objetos de la intervención médica. En este sentido, se pone de manifiesto la relación desigual y jerárquica que se establece entre las mujeres y el sistema sanitario, donde las mujeres aparecen como sujetos pasivos y dependientes frente a un equipo médico poseedor de un conocimiento experto:

En principio me explicaron lo que me iban a hacer, que me iban a quitar el pecho, que no me preocupara que luego habría este procedimiento del expansor y la prótesis. Y yo lo vi bien. Me informaron lo que era y todo el proceso que había, porque no es lo mismo que te quiten una parte a que te quiten entero. Entonces, bueno, bien. Es que era lo que había que hacer [sonríe] y ya está, es que tampoco te puedes plantear... (Mujer A).

\section{Durante el tratamiento}

Las zonas del cuerpo a las que hacen referencia durante el tratamiento, ocupando la centralidad de los discursos de la mayoría de las mujeres entrevistadas, son los pechos y el cabello. Sin embargo, llama la atención este dato, pues el tratamiento tiene consecuencias y efectos en otras partes del cuerpo. A este respecto, tan sólo una mujer hace alusión a zonas del cuerpo como la piel, las uñas o irrupciones cutáneas en nariz y boca:

Perdí la piel de las manos, de los pies, las uñas de los pies y de las manos, se me pusieron los ojos que no los podía abrir, la boca llena de llagas, la nariz toda llena 
de costras. Y sigo y sigo y empezó desde aquí, hasta ahí abajo. Pero bueno, ya pasó. (Mujer C).

En cuanto al pecho, cabe señalar que en él confluyen multitud de significados y experiencias relacionadas con la feminidad, pero también con la sexualidad y el placer. Sin embargo, la principal preocupación con respecto a la reconfiguración de los senos es el de ser menos atractivas para ellas mismas y para el otro, sólo citándose en uno de los casos la cuestión de la maternidad y, en ninguno, la pérdida de placer sexual en el pecho como zona erógena:

$Y$ hice así con mis manos - se toca el pecho- ¡Dios mío como estoy! Me vi esa cicatriz. El pecho este... (Mujer E).

Pese a ser una decisión sujeta a la elección de la paciente, la mayoría de las mujeres optan por la mastectomía radical. Las razones de ello son, en primer lugar, según refieren las propias mujeres, por tener una sensación de mayor tranquilidad al disminuir las probabilidades de que el tumor se reproduzca y; por otra, porque al optar por dicha opción, pueden sujetarse a una reconstrucción mamaria:

$Y$, es que, yo se lo dije al cirujano: yo no quiero medio, pecho. ¿Yo para qué quiero medio pecho? Me va a perjudicar psicológicamente más, medio trozo de carne, ahí, colgando, que si me lo quitan entero. Y tengo más tranquilidad. (Mujer A).

En cuanto al cabello, todas las mujeres entrevistadas hacen referencia al momento de la pérdida del pelo durante el tratamiento como uno de los momentos más impactantes vividos durante la enfermedad:

Es que... es muy contradictorio. Yo esperaba como el agua de mayo una caída de un pelo. Estaba más pendiente de eso que del malestar que tenía en realidad. (Mujer B).

La pérdida del cabello juega un papel muy importante en el estado emocional de las mujeres entrevistadas. Según Goffman (1986), detrás de este miedo puede estar por un lado, la preocupación por dejar de ser atractivas o deseables, cuestión esta central en la construcción de la identidad de género femenino hegemónica y, por otro, por el propio estigma de ser tratadas como personas enfermas, con cáncer:

La gente, por aquí, por el pueblo: ¡Hay que ver la melena que tienes, eh! A ti no se te cae el pelo. Y yo, a quien yo quería que supiera hasta el fondo de mi enfermedad, le decía: No es mi pelo, es una peluca. (Mujer G).

La confluencia de ambas pérdidas, seno y cabello, desarrolla en muchas de las mujeres sentimientos de baja autoestima y sensación de estar incompletas o "deformes":

$Y$ cuando estás con la quimio, por ejemplo, estás muy sensible porque aparte de que se te caiga el pelo, tú estás calva delante del espejo, sin un pecho, tú pareces un cuadro de Picasso, eres eso. Eso deforme eres tú. Pero tú tienes que ser muy fuerte para superar eso. (Mujer D). 


\section{Después del tratamiento}

Entre las opciones terapéuticas del tratamiento, se ofrece la posibilidad de prótesis mamaria con la finalidad de reconstruir la mama. Todas las mujeres entrevistadas optan por esta opción terapéutica, a excepción de una mujer dado que no se sometió a una mastectomía radical:

Hay mujeres que deciden no hacerse nada. Yo sería incapaz. Yo no podría verme al espejo... que no pasa nada, no pasa nada porque yo me he estado mirando al espejo y he tenido un pecho si y uno no, pero bueno, tenía la cosa de que... (Mujer F).

Pese a esta decisión voluntaria, y una vez que ha sido puesta la prótesis, las mujeres en sus relatos expresan como no sienten como propio el nuevo seno reconfigurado. Algunas de ellas, ponen de relieve la importancia, y por tanto la carencia, del pezón y la areola, en tanto en cuanto son símbolos característicos de esta zona, sin los cuales el pecho pierde significado:

$Y$ sin embargo por ponerme una prótesis, que no tengo yo un pecho en condiciones, porque... eso ni tiene pezón, ni tiene nada, ni nada, ni nada. (Mujer A).

Tan solo una mujer manifiesta expresamente haber tenido dudas a la hora de tomar la decisión de implantarse la prótesis mamaria. Finalmente, esta mujer narra que accede por la presión recibida desde el entorno y desde el propio sistema sanitario; lo que pone de relieve el férreo control que sobre los cuerpos de las mujeres impone la exigencia social de cumplir con los cánones estéticos normativos de belleza femenina:

Yo tengo una amiga que ella dice: "Dios mío, a mi me pasa lo que a ti y yo me quedo sin comer, me quedo... Por ponerme el pecho". (Mujer E).

Ahora bien, esta opción terapéutica del tratamiento causa insatisfacción en la mayoría de las mujeres entrevistadas dados los problemas que ha generado el implante mamario. En muchos casos se ha producido un encapsulamiento que, en el peor de los casos, dificulta el desarrollo de las tareas de la vida cotidiana:

Porque yo lo tengo aquí día a día, que me duele, que me jode y que yo no puedo hacer mi vida. Porque, a mí, un pecho no me sirve para nada para yo trabajar, para yo llevar mi casa para adelante, pero un brazo sí. (Mujer C).

\section{El cuerpo y la dimensión de la interacción}

La relación que las mujeres entrevistadas establecen con su cuerpo se ve muy influenciada por el vínculo, la aceptación y la validación del otro. Si las mujeres experimentan sentimientos de pérdida al ver su reflejo en el espejo, la mirada ajena se vive, en muchas ocasiones, como un señalamiento. Una de las mujeres relata el rechazo de su marido a ver las marcas que la enfermedad había dejado en su cuerpo:

Es que mi marido tiene muy poco espíritu. Mi marido tardó en verme por lo menos 5 meses, la herida, porque no era capaz. No era capaz. Y yo lo respetaba. Pero ya le dije: "¿Tú no puedes pensar que yo piense que te doy asco?". (Mujer C). 
Otras manifiestan sentir vergüenza para desnudarse delante de sus maridos, temerosas de mostrar su nueva reconstrucción corporal:

Hombre yo... al principio cuando me metía en la ducha yo no quería que mi marido entrara. Siempre me ponía de espaldas. (Mujer F).

Por ello, las mujeres ponen de relevancia el apoyo recibido por parte de su entorno en el proceso de aceptación de la nueva imagen corporal reconfigurada, resaltando fundamentalmente la figura de la pareja, buscando en ellos muestras de que siguen siendo atractivas:

Mi marido siempre diciéndome: "Niña, estás más guapa que antes, tienes otra cosa. Te han quitado una cosa y te han dado otra". Entonces, eso... Con eso ya... Tú te miras al espejo y te dices: "soy la mujer más guapa del mundo". (Mujer G).

\section{El cuerpo y la dimensión de las prácticas}

$\mathrm{Si}$ bien todas las mujeres manifiestan un sentimiento de pérdida muy importante a la hora de referirse a los efectos generados por el tratamiento, las estrategias de reconfiguración de su imagen corporal son diversas entre sí.

Sólo dos mujeres manifiestan haber usado peluca, pese a que todas ellas subrayaron como muy impactante el hecho de perder el cabello durante el tratamiento de quimioterapia. De ellas, una pone de relieve el hecho de pasar desapercibida en el pueblo en el que vive, de lo que se desprende un rechazo a ser tratada como enferma de cáncer de mama:

Y nada, yo me la compré, me la coloqué y... yo era la mujer más feliz del mundo porque nadie me lo notaba que yo llevaba una peluca puesta. Es que nadie me lo notaba, porque era de mí mismo color de pelo, mismo largo... (Mujer B).

El resto de las mujeres relatan haber usado pañuelos para esconder la alopecia y hacer su vida normal durante el tratamiento de quimioterapia, a excepción de los días en los que estaban más cansadas por los propios efectos generados por la quimio:

Cuando me puse por primera vez el pañuelo...Porque yo no he querido peluca. No lo he aceptado. La peluca para mí no... No, no, además es que ni tocarla, ¿no? No sé por qué, me dio... iNo! Mi pañuelo jy a la calle! (Mujer A).

El uso de tatuajes para esconder las marcas físicas ocasionadas por el tratamiento es mencionado por una de las mujeres:

Tengo un tatuaje hecho, pero ya de hace muchos años, que lo quería seguir... porque empieza aquí en la columna y termina aquí, justo debajo del pecho. Entonces ese tatuaje lo quería seguir para rodearme el pecho. (Mujer G).

Mientras que otra de las mujeres manifiesta haber pedido expresamente la posibilidad de borrar los puntos tatuados en el pecho durante la quimio, pues la hacían sentir marcada y estigmatizada: 
Me sentía como marcada, como si yo fuera una vaca llevando marcas, como diciendo "Yo me dado quimio, estoy operada" Que a mí no me importa porque yo lo cuento y yo... (Mujer D).

\section{DISCUSIÓN Y CONCLUSIONES}

A pesar de todas las zonas del cuerpo que se ven afectadas por el tratamiento del cáncer de mama, los principales hallazgos muestran la centralidad del cabello o de los senos en los discursos de las mujeres respecto a los efectos que el tratamiento ejerce sobre sus cuerpos en el plano subjetivo. Estos resultados concuerdan con otros estudios llevados a cabo en este campo de investigación (Belber-Gómez et al., 2018; Coll-Planas, 2014; Coll-Planas y Visa, 2015; Díaz, 2010; Núñez et al., 2018). En este sentido, la principal preocupación de las mujeres entrevistadas con respecto a los efectos del tratamiento del cáncer de mama sobre el cabello y los senos es la pérdida de atractivo. Motivo por el cual, la mayoría de las mujeres optan por implantarse una prótesis mamaria. Al igual que los resultados obtenidos en otros estudios, el nuevo seno reconfigurado genera insatisfacción en las mujeres (Díaz, 2010), y no llega a sentirse como propio (Coll-Planas, 2014; Coll-Planas y Visa, 2015).

Asimismo, los resultados manifiestan que para las mujeres entrevistadas en el proceso de aceptación de su nueva corporeidad es un elemento muy importante la validación «del otro» (Simmel, 1908, 1927). En gran parte ello es debido, tal y como se ha señalado en otros estudios (Díaz, 2010), porque sienten la mirada ajena en su nueva corporeidad como un señalamiento «del otro». De ahí que algunas mujeres tiendan a buscar muestras en su entorno para corroborar que aún siguen siendo atractivas y deseables (Wikilson, 2008). Por todo ello, las mujeres emprenden diferentes estrategias para reconfigurar su imagen corporal tras el tratamiento con la finalidad de disimular los efectos que sobre su cuerpo ha causado el mismo. Dichas estrategias van desde el uso de pelucas o pañuelos para esconder la alopecia, hasta la realización de tatuajes para disimular las cicatrices o los puntos de la radioterapia que, algunas mujeres, sienten como marcas que las estigmatizan.

\section{Limitaciones del estudio}

Si bien hemos obtenido un primer acercamiento a las vivencias de las mujeres durante el tratamiento del cáncer de mama en relación a sus cuerpos y la interacción con «el otro» (Simmel, 1908, 1927) no se ha explorado en profundidad las razones que llevan a estas mujeres a sentirse insatisfechas con su nueva corporeidad. Por tanto, dada la importancia de la mirada «del otro» en la construcción del cuerpo (Le Breton, 1990) y, por ende, en la propia imagen corporal; se hace imprescindible incorporar una «perspectiva interseccional» (Kimberlé, 1989) en investigaciones futuras. La perspectiva 
interseccional permite incluir categorías sociales como la clase social, la edad, nacionalidad, etnia e identidad de género que pueden tener una gran influencia, y ser explicativas, del vínculo que las mujeres con cáncer de mama establecen con sus cuerpos durante el proceso de la enfermedad.

\section{REFERENCIAS}

Belber, M., Valencia F., y De la Viuda, M.E. (2018). Análisis de la experiencia subjetiva en mujeres con cáncer de mama. Duazary: Revista Internacional de Ciencias de la Salud, 15(1), 71-85.

Biglia, N., Moggio, G., Peano, E., Sgandurra, P., Ponzone, R., Nappi, R., y Sismondi, P. (2010). Effects of surgical and adjuvant therapies for breast cancer on sexuality, cognitive functions, and body weight. Journal of Sexual Medicine, 7(5), 1891-1900.

Coll-Planas, G. (2014). «Me quedaré con lo positivo»: Análisis de blogs de mujeres con cáncer de mama. Aloma, 32(1), 33-44.

Coll-Planas, G., y Visa, M. (2015). La cicatriz (in) visible. La representación del cuerpo en blogs de mujeres con cáncer de mama. Política y Sociedad, 52(2), 487-507.

Crenshaw, K. (1989). Demarginalizing the Intersection of Race and Sex: A Black Feminist Critique of Antidiscrimination Doctrine, Feminist Theory and Antiracist Politics. Chicago: University of Chicago Law School.

Díaz, V.E. (2010). Sexualidad, cuerpo y duelo: experiencia clínica con mujeres diagnosticadas con cáncer ginecológico o de mama. Pensamiento Psicológico, 7(14), 155-160.

Durkheim, E. (1987). La división social del trabajo (Trad. C.G. Posada). Madrid: Akal.

Figueroa, M. (2018). La identidad "positiva" como violencia simbólica en mujeres con cáncer. GénEr웃 s, 24(22), 133-156.

Figueroa, M., Valadez, M., Rivera, M., y Montes, R. (2017). Evaluación de la imagen corporal en mujeres con cáncer de mama: una revisión sistemática. Universitas Psychologica, 16(4), 1-12. doi:10.11144/Javeriana.upsy16-4.eicm

Hammersley, M., y Atkinson, P. (1994). Etnografía. Métodos de Investigación. Barcelona: Paidós.

Le Breton, D. (1990). Anthropologie du corps et modernité. París: Presses universitaires de France.

Le Breton, D. (1991). Sociologie du corps: perspectives. París: Presses universitaires de France.

Núñez, C., Navarro, A., Cortés, S., López, J., Aguirre-Loaiza, H., y Trujillo, C. (2018). Imagen corporal (IC) y estrategias de afrontamiento (EA): Análisis de las características médico quirúrgicas en mujeres con diagnóstico de cáncer de mama. Terapia Psicológica, 36(2), 59-69.

Przezdziecki, A., Sherman, K., Baillie, A., Taylor, A., Foley, E., y Stalgis-Bilinski, K. (2013). My changed body: Breast cancer, body image, distress and self-compassion. Psychooncology, 22(8), 1872-1879.

Rodrigues, T., Ribeiro, M. y Tavares, M. S. (2012). Breast cancer repercussion in female body image: A systematic review. Physis, 22(3), 1003-1029. 
Rodríguez, C., Blasco, T., Vargas, A., y Benito, A. (2011). The Pertinence of Applying Qualitative Investigation Strategies in the Design and Evaluation of HIV Prevention Policies. En Y. Yi-Wei Tang (Ed.), Recent Translational Research in HIV/AIDS (pp. 123-157). USA: Intech.

Simmel, G. (1927). Sociología. Estudios sobre las formas de socialización (Trad. J. Pérez). Madrid: Revista de Occidente.

Teo, I., Reece, G.P., Christie, I.C., Guindani, M., Markey, M.K., Heinberg, L.J., ... y Fingeret, M.C. (2016). Body image and quality of life of breast cancer patients: influence of timing and stage of breast reconstruction. Psycho-Oncology, 25(9), 1106-1112.

Recibido: 20 de septiembre de 2019 Recepción Modificaciones: 10 de noviembre de 2019 Aceptado: 12 de noviembre de 2019 